\title{
RESSONÂNCIA MAGNÉTICA NA ESQUIZOFRENIA: UM ESTUDO MORFOMÉTRICO*
}

\author{
Cláudio Campi de Castro ${ }^{1}$
}

Resumo Trinta e três pacientes esquizofrênicos crônicos e 21 indivíduos normais foram submetidos a exames de ressonância magnética em aparelho de 1,5 T, sendo realizadas imagens ponderadas em T2 nos planos axial e coronal. Foram analisados, por métodos morfométricos semi-automáticos, os volumes intracraniano, supratentorial, infratentorial, do líquido cefalorraquiano total, ventricular e subaracnóide e do encéfalo. Foram ainda medidos os volumes do complexo amígdala-hipocampo, córtex do giro para-hipocampal, putâmen, globo pálido, lobo temporal e substâncias branca e cinzenta do lobo temporal. Foram calculados os volumes relativos dessas estruturas, corrigidos pelo volume intracraniano. As alterações mais relevantes observadas nos esquizofrênicos, em comparação com os controles, foram de redução do volume relativo do encéfalo, aumento do volume relativo do líquido cefalorraquiano ventricular, subaracnóide e total. Foi ainda observada redução do volume dos complexos amígdala-hipocampo, dos lobos temporais e da substância branca dos lobos temporais, e aumento de volume dos putâmens.

Unitermos: Esquizofrenia. Imagem por ressonância magnética. Morfometria.

\begin{abstract}
Magnetic resonance imaging in schizophrenia: a morphometric study.
Thirty-three patients with chronic schizophrenia and 21 normal subjects were submitted to magnetic resonance imaging studies using a $1.5 \mathrm{~T}$ scanner. Axial and coronal T2-weighted images were obtained. The volumes of the brain, intracranial, supratentorial, infratentorial and the total, ventricular and subarachnoid cerebrospinal fluid volumes were measured using semi-automated morphometric methods. The volumes of the amygdala-hippocampus complex, parahippocampal gyrus cortex, putamen, globus pallidus, temporal lobe , gray and white matter of temporal lobe were also measured. These volumes were normalized using the intracranial volume as reference. The most relevant findings observed were reduced brain volume and increased total, ventricular and subarachnoid cerebrospinal fluid volumes in patients with schizophrenia when compared to the controls. Patients with schizophrenia had also smaller amygdala-hippocampus complexes, temporal lobes and temporal lobe white matter than the controls, as well as increased putamen volumes. Key words: Schizophrenia. Magnetic resonance imaging. Morphometry.
\end{abstract}

\section{INTRODUÇÃO}

O Manual Estatístico e Diagnóstico de Distúrbios Mentais da Associação Psiquiátrica Americana define, como características principais da esquizofrenia, a presença de sintomas psicóticos peculiares na fase ativa da doença e funcionamento abaixo do nível mais alto previamente atingido ${ }^{(\mathbf{1})}$. Este manual determina que o diagnóstico é feito apenas quando não se é possível estabelecer um fator orgânico que tenha iniciado e mantido a doença. No entanto, vários pesquisadores tentam, há décadas, encontrar um subs-

* Estudo realizado no Centro de Pesquisa Psiquiátrica Ambulatorial e no Serviço de Ressonância Magnética da Universidade da Califórnia, San Diego, Califórnia, EUA.

1. Médico Chefe da Seção de Ressonância Magnética do Instituto do Coração (InCor), Hospital das Clínicas da Faculdade de Medicina da Universidade de São Paulo.

Endereço para correspondência: Dr. Cláudio Campi de Castro. Rua José Maria Lisboa, 1168, apto. 102. São Paulo, SP, 01423-001. E-mail: campi@uol.com.br

Aceito para publicação em 10/4/2001 trato anatômico ou bioquímico para esta doença.

Grande número de trabalhos de anatomia patológica foi publicado, descrevendo diversas alterações em encéfalos de cadáveres de indivíduos esquizofrênicos submetidos a autópsia. $\mathrm{O}$ advento da tomografia computadorizada trouxe novo impulso à pesquisa de alterações anatômicas em esquizofrênicos, por permitir um estudo, in vivo, não-invasivo do encéfalo e dos espaços preenchidos por líquido cefalorraquiano. Entretanto, a baixa capacidade de caracterização tecidual, o uso de cortes relativamente espessos e a dificuldade de realização de cortes em diversos planos não permitiu à tomografia computadorizada um estudo mais refinado da anatomia do encéfalo dos esquizofrênicos, restringindo-se à constatação de aumento ventricular e alargamento de sulcos corticais ${ }^{(2)}$.

A ressonância magnética revolucionou o estudo in vivo da morfologia do encélalo de pacientes esquizofrênicos. A possibilidade de se realizar cortes em qualquer orientação desejada e a excelente diferenciação entre as substâncias branca e cinzenta proporcionaram à ressonância magnética a capacidade de se gerar imagens do encéfalo com uma riqueza de detalhes inigualada por outros métodos, aproximando-se à de um corte de peça anatômica, sem o inconveniente da radiação ionizante produzida pela tomografia computadorizada.

A ressonância magnética é um método que está em plena evolução no momento atual. Duas novas técnicas, a espectroscopia por ressonância magnética e a ressonância magnética funcional, permitem, atualmente, o estudo do metabolismo encefálico in vivo.

Diversos trabalhos foram realizados com a utilização da ressonância magnética para tentar-se comprovar a existência de alterações estruturais encefálicas em esquizofrênicos. Assim, há trabalhos 
de ressonância magnética em que se estuda o volume intracraniano e do encéfalo, volume dos ventrículos, do espaço subaracnóide, área do corpo caloso, volume dos lobos frontais, parietais e occipitais, lobos temporais e complexos amígdala-hipocampo, núcleos da base e tálamos, tronco cerebral e hemisférios cerebelares. Estudaram-se ainda alterações no septo pelúcido, presença de cavos do septo pelúcido e de Verga ${ }^{(3-6)}$.

Atualmente, vários trabalhos de ressonância magnética funcional têm sido realizados em esquizofrênicos ${ }^{(7,8)}$.

O objetivo deste trabalho é investigar a existência de possíveis alterações morfológicas de estruturas intracranianas em pacientes portadores de esquizofrenia, utilizando-se um método morfométrico por ressonância magnética.

\section{MATERIAL E MÉTODOS}

Os pacientes foram selecionados do Centro de Pesquisa Psiquiátrica Ambulatorial da Universidade da Califórnia, San Diego, EUA, conforme metodologia citada por Jernigan et al. ${ }^{(\mathbf{9})}$. Todos os pacientes satisfaziam os critérios de esquizofrenia pelo DSM-III-R ${ }^{(\mathbf{1})}$. O diagnóstico foi baseado no Structured Clinical Interview for DSM-III-R (SCID-P) ${ }^{(\mathbf{1 0})}$. Os pacientes foram excluídos do trabalho se tinham história de doença neurológica ou de outra etiologia, incluindo perda de consciência significativa por qualquer causa. Todos os pacientes esquizofrênicos estavam em uso ou haviam feito uso de neurolépticos no passado.

Trinta e três pacientes esquizofrênicos crônicos e 21 indivíduos normais foram submetidos a exames de ressonância magnética em aparelho de 1,5 $\mathrm{T}$, no Serviço de Ressonância Magnética da Universidade da Califórnia, sendo realizadas imagens ponderadas em T2 nos planos axial e coronal, primeiro e segundo ecos, com tempo de repetição de $2.000 \mathrm{~ms}$, tempos de eco de 25 e $70 \mathrm{~ms}$, espessura de corte de $5 \mathrm{~mm}$, intervalo entre cortes de $2,5 \mathrm{~mm}$, matriz de $256 \times$ 256 , campo de visão de $24 \mathrm{~cm}$ e duas excitações. As imagens foram processadas por computador e foram submetidas a métodos morfométricos semi-automáticos para medida de volume de diversas estruturas intracranianas ${ }^{(9)}$. Foram analisados os volumes intracraniano, supratentorial, infratentorial, do líquido cefalorraquiano total, ventricular e subaracnóide e do encéfalo. Foram ainda medidos, manualmente, os volumes do com- plexo amígdala-hipocampo, córtex do giro para-hipocampal, putâmen, globo pálido, lobo temporal e, de maneira semiautomática, foram segmentadas e calculados os volumes das substâncias branca e cinzenta do lobo temporal (Figuras 1 e 2). Foram calculados os volumes relativos dessas estruturas, corrigidos pelo volume intracraniano.

Os dados foram avaliados pelos testes $t$ de Student, Mann-Whitney, qui-quadrado e análise de perfis, quando indicados, com nível de significância de 0,05 e testes bicaudais.

\section{RESULTADOS}

O grupo controle foi constituído por cinco mulheres e 16 homens, e o grupo de pacientes esquizofrênicos, por $12 \mathrm{mu}$ lheres e 21 homens, não havendo diferença significativa na composição dos grupos quanto ao sexo ( $\mathrm{p}>0,32)$. Quanto à idade dos indivíduos, o grupo controle mostrou idade média de 32,4 anos e desvio padrão de 6,7 anos $(n=21)$, e o grupo de esquizofrênicos, idade média de 30,7 anos e desvio padrão de 7,8 anos ( $n=33)$, não havendo diferença significativa entre os grupos quanto às médias de idade $(\mathrm{p}>0,21)$.

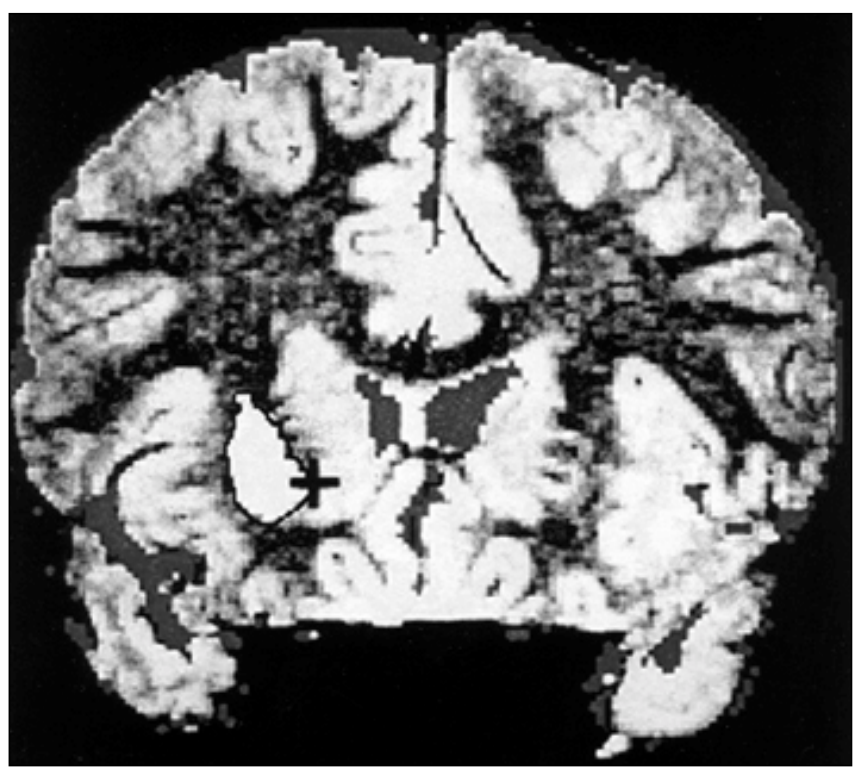

Figura 1. Imagem de ressonância magnética, corte coronal, após processamento em computador por programa de segmentação semi-automática. 0s "pixels" estão segmentados em substância branca, cinzenta e líquido cefalorraquiano. 0 operador traça uma região de interesse em torno do putâmen direito, com o auxílio de um cursor em forma de cruz.

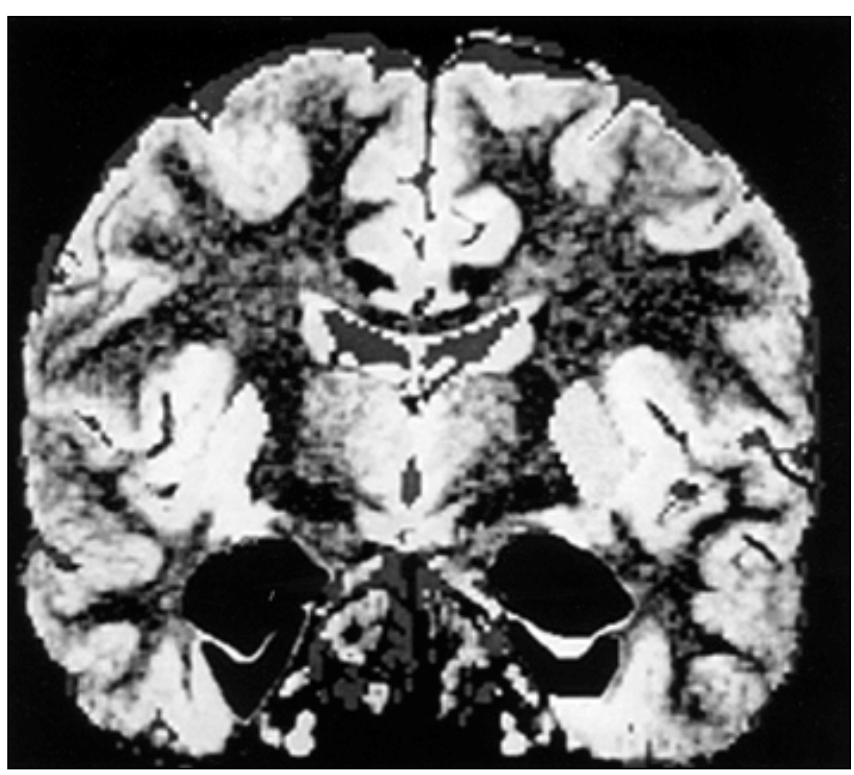

Figura 2. Corte coronal posterior ao da Figura 1, após a medida da área do complexo amígdala-hipocampo e córtex do giro para-hipocampal, bilateralmente. 
Na Tabela 1 são mostrados o volume intracraniano, em $\mathrm{cm}^{3}$, e os volumes relativos do líquido cefalorraquiano total, ventricular e subaracnóide, bem como o volume relativo encefálico, de controles e esquizofrênicos.

Observa-se que o volume intracraniano foi semelhante nos dois grupos estudados, ao passo que os volumes relativos do líquido cefalorraquiano total, ventricular e subaracnóide foram maiores nos pacientes esquizofrênicos; no entanto, o volume relativo encefálico foi menor nos esquizofrênicos, indicando uma redução volumétrica encefálica nestes pacientes, com conseqüente aumento dos espaços liquóricos.

Na Tabela 2 são mostrados os volumes relativos (porcentuais), corrigidos pelo volume intracraniano, dos complexos amígdala-hipocampo, córtex dos giros para-hipocampais, putâmens, globos pálidos, lobos temporais e substâncias branca e cinzenta dos lobos temporais.

$\mathrm{O}$ efeito de interação entre os grupos de esquizofrênicos e entre os lados direito e esquerdo foi não-significante para todas as variáveis. Os esquizofrênicos apresentaram, em relação aos controles, complexos amígdala-hipocampo, lobos temporais e substância branca dos lobos temporais menores que os dos controles. Por outro lado, os putâmens se apresentaram de maior volume nos esquizofrênicos que nos controles. Não houve diferença significativa entre os grupos quanto ao córtex do giro para-hipocampal e substância cinzenta dos lobos temporais.

Tabela 1 Volume absoluto intracraniano $\left(\mathrm{em}^{3} \mathrm{~cm}^{3}\right.$ ), volume relativo encefálico e do líquido cefalorraquiano (LCR) de indivíduos controles e pacientes esquizofrênicos.

\begin{tabular}{|l|r|r|r|r|l|}
\hline \multirow{2}{*}{ Volumes } & \multicolumn{2}{|c|}{$\begin{array}{c}\text { Controles } \\
(\mathrm{n}=21)\end{array}$} & \multicolumn{2}{c|}{$\begin{array}{c}\text { Esquizofrênicos } \\
(\mathrm{n}=33)\end{array}$} & \multirow{2}{*}{$\mathrm{p}$} \\
\cline { 2 - 5 } & \multicolumn{1}{|c|}{ Média } & \multicolumn{1}{|c|}{ DP } & Média & \multicolumn{1}{c|}{ DP } & \\
\hline Intracraniano $\left(\mathrm{cm}^{3}\right)$ & $1.470,97$ & 141,03 & $1.461,92$ & 142,23 & 0,41 \\
LCR total (\%) & 7,53 & 2,34 & 9,72 & 2,72 & $0,002^{*}$ \\
LCR ventricular (\%) & 1,23 & 0,50 & 1,52 & 0,53 & $0,02^{*}$ \\
LCR subaracnóide (\%) & 6,30 & 1,95 & 8,20 & 2,39 & $0,002^{*}$ \\
Encefálico (\%) & 92,47 & 2,34 & 90,28 & 2,72 & $0,002^{*}$ \\
\hline
\end{tabular}

DP, desvio padrão; ${ }^{*}$, diferença estatisticamente significativa.

Volume relativo de determinado parâmetro $=$ volume do parâmetro $\left(\mathrm{em} \mathrm{cm}^{3}\right) \times 100 /$ volume intracraniano $\left(\mathrm{em} \mathrm{cm}^{3}\right)$.
Quanto à análise de lado direito $\times$ esquerdo, houve diferença significativa quanto às variáveis complexo amígdalahipocampo, putâmen, globo pálido, lobo temporal e substância branca do lobo temporal.

\section{DISCUSSÃO}

Há décadas tenta-se encontrar um substrato anatômico para as patologias psiquiátricas, especialmente a esquizofrenia. Vários estudos de anatomia patológica encontraram alterações no encéfalo de esquizofrênicos, principalmente relacionadas a atrofia cortical e dilatação ventricular. Com o advento dos métodos de diagnóstico por imagem, pôde-se estudar, in vivo, possíveis alterações morfológicas no encéfalo dos esquizofrênicos. Estudos iniciais com pneumoencefalografia e, posteriormente, com tomografia computadorizada confirmaram os achados de atrofia cortical e dilatação ventricular $^{(2)}$.

A utilização clínica da ressonância magnética permitiu grande avanço na avaliação in vivo e de modo não-invasivo dos aspectos neuroanatômicos de pacientes portadores de patologias psiquiátricas. Desde a metade dos anos 80 a maior parte dos trabalhos de imagem

Tabela 2 Volume de estruturas do sistema límbico, núcleos da base e lobos temporais de indivíduos controles e pacientes esquizofrênicos, em relação ao volume intracraniano.

\begin{tabular}{|c|c|c|c|c|c|c|}
\hline \multirow[t]{2}{*}{ Volumes } & \multicolumn{2}{|c|}{$\begin{array}{c}\text { Controles } \\
(\mathrm{n}=21)\end{array}$} & \multicolumn{2}{|c|}{$\begin{array}{c}\text { Esquizofrênicos } \\
(n=33)\end{array}$} & \multicolumn{2}{|c|}{$\mathrm{p}$} \\
\hline & Média & $\mathrm{DP}$ & Média & DP & $\mathrm{D} \times \mathrm{E}$ & $\mathrm{C} \times \mathrm{E}$ \\
\hline Complexo amígdala-hipocampo direito & 4,76 & 0,44 & 4,47 & 0,53 & $000009 *$ & $00289 *$ \\
\hline Complexo amígdala-hipocampo esquerdo & 4,51 & 0,38 & 4,32 & 0,39 & 0,0009 & $0,0<89$ \\
\hline Córtex do giro para-hipocampal direito & 1,29 & 0,28 & 1,22 & 0,31 & 0.4277 & 0.5052 \\
\hline Córtex do giro para-hipocampal esquerdo & 1,32 & 0,33 & 1,30 & 0,31 & & \\
\hline Putâmen direito & 4,07 & 0,35 & 4,33 & 0,49 & $00130 *$ & $00142 *$ \\
\hline Putâmen esquerdo & 4,12 & 0,31 & 4,45 & 0,48 & & \\
\hline Globo pálido direito & 1,83 & 0,29 & 1,82 & 0,31 & $0,0054^{*}$ & 0.6036 \\
\hline Globo pálido esquerdo & 1,68 & 0,28 & 1,77 & 0,32 & 0,0054 & 0,0030 \\
\hline Lobo temporal direito & 44,60 & 4,85 & 42,09 & 4,70 & $0,0330 *$ & $00132 *$ \\
\hline Lobo temporal esquerdo & 43,55 & 4,64 & 40,10 & 5,10 & & \\
\hline Lobo temporal direito (substância branca) & 16,61 & 3,61 & 14,49 & 3,70 & $0,0007^{*}$ & $0,0112^{*}$ \\
\hline Lobo temporal esquerdo (substância branca) & 15,72 & 3,77 & 12,89 & 3,33 & 0,0001 & \\
\hline Lobo temporal direito (substância cinzenta) & 28,00 & 3,71 & 27,60 & 3,86 & 0.5812 & 0.5561 \\
\hline Lobo temporal esquerdo (substância cinzenta) & 27,83 & 3,01 & 27,21 & 3,50 & & \\
\hline
\end{tabular}

$D P$, desvio padrão; $D \times E$, comparação entre os lados direito e esquerdo; $C \times E$, comparação entre os grupos de indivíduos controles e esquizofrênicos; ${ }^{*}$, diferença estatisticamente significativa.

Volume relativo de determinado parâmetro $=$ volume do parâmetro $\left(\mathrm{em}_{\mathrm{cm}}^{3}\right) \times 100 /$ volume intracraniano $\left(\mathrm{em} \mathrm{cm}^{3}\right)$. 
sobre patologias psiquiátricas passou a utilizar a ressonância magnética, devido à sua capacidade de realizar cortes em diversos planos, com alta resolução espacial e com capacidade de caracterização tecidual superior aos outros métodos ${ }^{(\mathbf{6})}$.

Quanto ao volume do líquido cefalorraquiano, vários estudos demonstraram aumento do volume dos ventrículos, bem como aumento do volume do espaço subaracnóide ${ }^{(3-6)}$. Nossos achados demonstraram, em concordância com os da literatura, aumento do volume do sistema ventricular, aumento do volume do líquido cefalorraquiano subaracnóide (secundário provavelmente a atrofia cortical) e aumento global do volume do líquido cefalorraquiano intracraniano. Estas observações, associadas à ausência de diferença significativa entre os grupos controle e de esquizofrênicos, quanto ao volume intracraniano, leva a crer que existe uma redução de volume do encéfalo dos esquizofrênicos em alguma fase da sua evolução, quando comparados aos indivíduos controles.

A redução de volume dos lobos temporais e dos complexos amígdala-hipocampo é achado freqüentemente relata$\mathrm{do}^{(6,11)}$, e também por nós observado. A redução volumétrica dos lobos temporais e complexos amígdala-hipocampo se deu principalmente à esquerda nos nossos pacientes, acompanhada de redução do volume da substância branca do lobo temporal, que também é corroborado por dados da literatura ${ }^{(\mathbf{1 2})}$.

Interessante é a observação de aumento de volume dos putâmens em nossos pacientes. Inicialmente visto como achado curioso ${ }^{(9)}$, foi posteriormente comprovado por vários autores, devendo-se provavelmente ao uso de antipsicóticos típicos, bloqueadores dos receptores centrais de dopamina (D2). Assim, pacientes em uso de psicóticos típicos apresentam aumento do volume dos núcleos caudado e putâmen, havendo redução de volume destes núcleos quando são utilizados neurolépticos atípicos (com pouco efeito de bloqueio de receptores de dopamina) como clozapina ${ }^{(6,13-17)}$.

\section{CONCLUSÕES}

Conclui-se que existem alterações morfológicas de estruturas intracranianas em esquizofrênicos crônicos, quando comparados a indivíduos normais, detectáveis por meio de análise morfométrica de imagens de ressonância magnética.

As alterações mais relevantes observadas nos esquizofrênicos, em comparação com os controles, foram redução do volume relativo do encéfalo, aumento do volume relativo do líquido cefalorraquiano ventricular, subaracnóide e total. Foi ainda observada redução do volume dos complexos amígdala-hipocampo, dos lobos temporais e da substância branca dos lobos temporais, e aumento de volume dos putâmens, este último achado provavelmente relacionado ao uso de neurolépticos.

\section{Agradecimentos}

Agradecemos aos Drs. John R. Hesselink e Terry Jernigan, da Universidade da Califórnia, San Diego, EUA, por permitir acesso às imagens e dados clínicos dos pacientes e fornecer a infra-estrutura computacional para a realização deste trabalho, e ao Dr. Ismar Bancovsky, pela orientação na realização deste trabalho. Agradecemos ainda a Sarah Archibald e David Foster, do Brain Image Analysis Laboratory, da Universidade da Califórnia, pelo armazenamento e pré-processamento das imagens e dados clínicos dos pacientes e controles.

\section{REFERÊNCIAS}

1. American Psychiatric Association. Diagnostic and statistical manual of mental disorders. 3rd ed. Washington, DC: American Psychiatric Association, 1987.

2. Krishnan KRR, Boyko OB, Figiel GS. Imaging in psychiatric disorders. In: Greenberg JO, ed. Neuroimaging: a companion to Adams and Victor's
Principles of neurology. New York: Mc Graw-Hill, 1994:227-49.

3. Stratta P, Mancini F, Mattei P, Daneluzzo E, Casacchia M, Rossi A. Association between striatal reduction and poor Wisconsin card sorting test performance in patients with schizophrenia. Biol Psychiatry 1997;42:816-20.

4. McCarley RW, Shenton ME, O'Donnell BF, Nestor PG. Uniting Kraepelin and Bleuler: the psychology of schizophrenia and the biology of temporal lobe abnormalities. Harv Rev Psychiatry 1993;1:36-56.

5. Wright IC, McGuire PK, Poline JB, et al. A voxelbased method for the statistical analysis of gray and white matter density applied to schizophrenia. Neuroimage 1995;2:244-52.

6. Buckley PF. Structural brain imaging in schizophrenia. Psychiatr Clin North Am 1998;21:77-92.

7. Weinberger DR, Mattay V, Callicott J, et al. fMRI applications in schizophrenia research. Neuroimage 1996;4(suppl 3):S118-26.

8. Bertolino A, Kumra S, Callicott JH, et al. Common pattern of cortical pathology in childhoodonset and adult-onset schizophrenia as identified by proton magnetic resonance spectroscopic imaging. Am J Psychiatry 1998;155:1376-83.

9. Jernigan TL, Zisook S, Heaton RK, Moranville JT, Hesselink JR, Braff DL. Magnetic resonance imaging abnormalities in lenticular nuclei and cerebral cortex in schizophrenia. Arch Gen Psychiatry 1991;48:881-90.

10. Spitzer RL, Williams JBW, Gibbon M, First MB. Instruction manual for the structured clinical interview for DSM-III-R (SCID). New York: New York State Psychiatric Institute, Biometrics Research, 1989.

11. Bryant NL, Buchanan RW, Vladar K, Breier A, Rothman M. Gender differences in temporal lobe structures of patients with schizophrenia: a volumetric MRI study. Am J Psychiatry 1999; 156:603-9.

12. Sigmundsson T, Suckling J, Maier M, et al. Structural abnormalities in frontal, temporal, and limbic regions and interconnecting white matter tracts in schizophrenic patients with prominent negative symptoms. Am J Psychiatry 2001;158:234-43.

13. Chakos MH, Lieberman JA, Bilder RM, et al. Increase in caudate nuclei volumes of first-episode schizophrenic patients taking antipsychotic drugs. Am J Psychiatry 1994;151:1430-6.

14. Chakos MH, Lieberman JA, Alvir J, Bilder R, Ashtari M. Caudate nuclei volumes in schizophrenic patients treated with typical antipsychotics or clozapine. Lancet 1995;345:456-7.

15. Frazier JA, Giedd JN, Kaysen D, et al. Childhoodonset schizophrenia: brain MRI rescan after 2 years of clozapine maintenance treatment. Am J Psychiatry 1996;153:564-6.

16. Busatto GF, Kerwin RW. Schizophrenia, psychosis, and the basal ganglia. Psychiatr Clin North Am 1997;20:897-910.

17. Corson PW, Nopoulos P, Miller DD, Arndt S, Andreasen NC. Change in basal ganglia volume over 2 years in patients with schizophrenia: typical versus atypical neuroleptics. Am J Psychiatry 1999;156:1200-4. 